\title{
The role of guessing and boundaries on date estimation biases
}

\author{
PETER JAMES LEE and NORMAN R. BROWN \\ University of Alberta, Edmonton, Alberta, Canada
}

\begin{abstract}
This study investigates the causes of event-dating biases. Two hundred participants provided knowledge ratings and date estimates for 64 news events. Four independent groups dated the same events under different boundary constraints. Analysis across all responses showed that forward telescoping decreased with boundary age, concurring with the boundary-effects model. With guesses removed from the data set, backward telescoping was greatly reduced, but forward telescoping was unaffected by boundaries. This dissociation indicates that multiple factors (e.g., guessing and reconstructive strategies) are responsible for different dating biases and argue against a boundary explanation of forward telescoping.
\end{abstract}

The study of real-world estimation has demonstrated that people often use multiple strategies when generating responses (Brown, 2002). This research has also shown that strategy selection is related to the strength and direction of estimation bias. For example, when estimating event frequencies, enumeration strategies (retrieving and counting instances) can result in underestimation because forgotten items are excluded (Blair \& Burton, 1987; Brown, 1995; Brown \& Sinclair, 1999). Nonetheless, it is uncommon for researchers to consider their data as the product of a number of discrete strategies associated with different types of bias. This can lead to misinterpretation because averaging over strategies can obscure the true causes of estimation biases (Siegler, 1987a).

In the present article, we apply this multiple-strategies approach to a well-studied real-world task - date estimation. We demonstrate that an accurate understanding of people's estimation strategies, and associated biases, allows us to compare two very different models of event dating - the boundary-effects model (Huttenlocher, Hedges, \& Prohaska, 1988; Rubin \& Baddeley, 1989) and the associative model (Kemp, 1999). We do this by examining the effects of boundaries on dating bias when guessed and nonguessed responses are taken into account.

There are at least three strategies for estimating event dates: People can retrieve dates directly from long-term memory (Friedman, 1993; Larsen, Thompson, \& Hansen, 1996); they can access relevant domain-specific knowledge to infer the date (Brown, 1990; Friedman, 1993; Thompson, Skowronski, \& Betz, 1993); or they can guess (Brown, 2002; Thompson, Skowronski, \& Lee, 1988). Knowledge-based temporal estimates, and the pro-

This research was supported by a NSERC Operating Grant 138076 awarded to N.R.B. Correspondence concerning this article should be sent to P. J. Lee, Department of Psychology, University of Alberta, Edmonton, AB, T6G 2E9 Canada (e-mail: pjlee@ualberta.ca). cesses that produce them, have been extensively studied (for reviews, see Friedman, 1993; Thompson, Skowronski, Larsen, \& Betz, 1996). In contrast, the systematic examination of direct retrieval and guessing have been almost completely ignored by researchers interested in eventdating biases (see, however, Burt, 1992; Burt, Kemp, \& Conway, 2001; Thompson et al., 1988). It appears that direct retrieval has been overlooked because it is uncommon (Brown, Rips, \& Shevell, 1985; Friedman, 1993) and that guessing has been neglected because it is assumed to produce estimates that are inaccurate but unbiased.

Understanding guessing may be important because guesses are not necessarily random answers. For instance, when people guess populations for unfamiliar countries, their estimates are often much lower than for known countries (Brown, 2002). This is because people assume that unknown countries are obscure and have small populations. Respondents can still be quite accurate because poorly known countries often have smaller populations. People have also been shown to use lack of knowledge to determine the likelihood that an assertion is either true or false (Gentner \& Collins, 1981) and to make accurate paired comparison judgments by selecting known items over unknown items (Goldstein \& Gigerenzer, 1999). Of course, people's assumptions are not always valid, and guessing may lead to biases.

Lack of knowledge does not inevitably prohibit people from answering, because other information (i.e., response ranges) may be useful when they generate plausible responses. However, researchers often treat guessing as an unexplained and unbiased error rather than a cause of systematic estimation bias. As a result, guessing may misrepresent the true pattern of responses because the effects of guessing may go unnoticed. Here, we demonstrate the importance of discriminating between guessed and nonguessed responses by showing that aggregating over response strategies can confound evaluation of the boundaryeffects and associative models. In the remainder of this 
section, we describe temporal estimation biases, provide details for each theoretical account, and present a dualstrategy perspective that compares these approaches.

Two biases are observed when people estimate the date for past events. Forward telescoping refers to a tendency to date events as more recent than their actual dates (Baddeley, Lewis, \& Nimmo-Smith, 1978; Brown et al., 1985; Huttenlocher et al., 1988; Rubin \& Baddeley, 1989; Thompson, 1982). This bias tends to increase in magnitude with event age and typically affects older events. In contrast, events that are estimated as older than their actual dates are referred to as backward telescoped. Researchers often find that recent events tend to be backward telescoped (e.g., Crawley \& Pring, 2000; Ferguson \& Martin, 1983; Rubin \& Baddeley, 1989). Both the boundary-effects and associative models attempt to explain telescoping effects from the standpoint of single underlying causes, though they do so from very different perspectives.

\section{The Boundary-Effects Approach}

Here, boundaries refer to absolute limits on people's responses. For example, asking the question "Since January 1997, when did...?" limits estimates to dates after the boundary. The boundary-effects model (Huttenlocher et al., 1988; Rubin \& Baddeley, 1989) is predicated on three assumptions. First, without boundaries, mean estimated dates are unbiased, although some variability among people's responses is assumed (i.e., a distribution of error around the mean). Second, the amount of variability increases as the target events become older because memory for dates is likely to decrease over time. Third, the model assumes that boundaries serve to truncate the distribution of errors on one side. This predicts error distributions with one tail trimmed because estimates beyond the boundaries are disallowed or because the respondent declines to date events thought to be older than the boundary. The boundary-effects model predicts the typical pattern of telescoping effects because the truncation of error distributions shifts the mean value away from the boundary. As error variance increases with event age, the magnitude of the bias also increases. Backward telescoping can be explained by the same mechanism because the present acts as a boundary and past events cannot have occurred in the future.

Although the boundary-effects perspective has been shown to describe biases in a number of studies (Burt, 1992; Huttenlocher et al., 1988; Rubin \& Baddeley, 1989), other results have not been encouraging. Ferguson and Martin (1983), Kemp (1993), and Lieury, Caplain, Jacquet, and Jolivet (1979) have demonstrated that forward telescoping occurs in the absence of any explicit boundaries. These results suggest that although boundaries may affect response bias, they may not be the primary cause of telecoping effects.

\section{The Associative Approach}

The associative model (Kemp, 1999) is an attempt to explain forward telescoping without recourse to boundaries. This position assumes that inferring dates involves memory for better-known events that are similar and contextually related to the target item. When a suitable associate is retrieved, the date for this event serves as the best estimate for the date of the target event. Because the ability to retrieve relevant information is likely to decrease over time, the date of the associated event is likely to be more recent than that of the target; associations that can be constructed will be more frequent when closer to the present, resulting in a tendency to forward telescope
(A) BoundaryEffects Model: All Responses

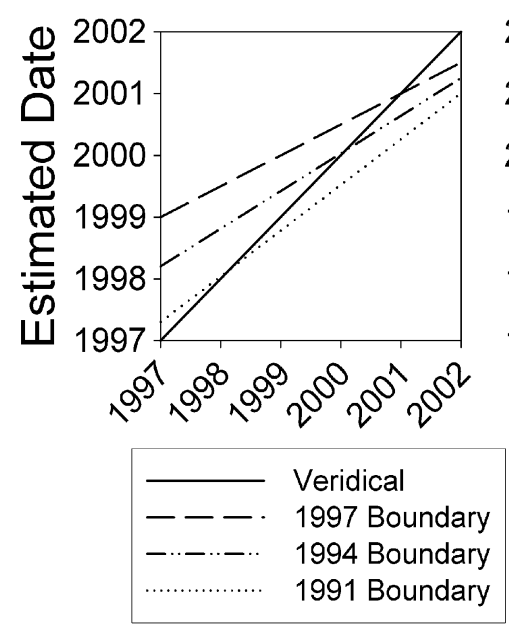

(B) Associative

Model: Nonguessed Responses

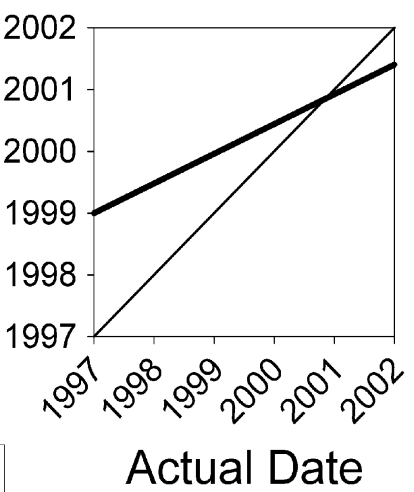

(C) Guessed

Responses

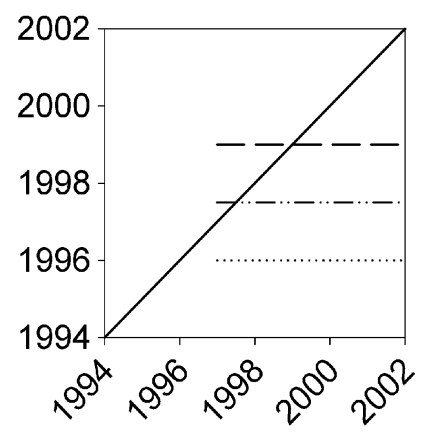

Figure 1. Predicted effects of boundaries on response biases for the boundary-effects and associative models under differing boundary conditions. 
estimates. However, if the respondent has never heard of an event, few systematic inferences can be made. Thus, the associative model does not prescribe how people guess and makes no predictions about the biasing of guessed responses.

\section{A Dual-Strategies Perspective}

The critical difference between the boundary-effects and associative positions is the predicted effect of boundaries on estimation biases. Here, we compare the two models by asking participants to date the same events, but with different boundary limitations given to separate groups of respondents. The boundary-effects model predicts a very different pattern of response biases than the associative model does. The boundary-effects model predicts that for nonguessed responses, forward telescoping should decrease as boundaries become more remote because of a decrease in the truncation of error distributions. In addition, the model predicts that the amount of backward telescoping for recent events will remain the same because the truncation of error distributions by the present (i.e., time of study) should remain constant for recent events, irrespective of early boundaries. The critical prediction is a significant boundary $X$ actual date interaction for nonguessed responses (Figure 1A). In contrast, the associative model predicts that there should be no effect of boundaries on nonguessed responses when the same events are dated (Figure 1B). For nonguessed responses, the model predicts a main effect of actual date, but no main effect of boundaries and no interaction.

These predictions refer only to nonguessed responses, because boundary information may be especially relevant when people guess. When an event is unknown or unrecognized, temporal information is likely to be sparse or nonexistent, although the response range can be easily calculated using boundaries. We hypothesize that people estimate dates for unknown events in two ways, both of which involve boundary information. First, respondents might provide estimates close to the earliest boundary on the assumption that unrecognized events were once known but have since been forgotten and that older events are more likely to have been forgotten than more recent events. Thus, unrecognized events are likely to be old (Brown et al., 1985). Second, they can estimate the middle of a bounded range because this type of response will reduce absolute error (Huttenlocher, Hedges, \& Duncan, 1991; Thompson et al., 1988).

Pilot research indicated that the number of unknown events in the stimuli used here was not correlated with event age. Holding the proportion of guesses uniform across each year allowed us to examine the effect of guessing strategies as a constant. This approach simplified our comparison of each model when all data were analyzed, and with guesses removed from the data set. The predicted effect of boundaries on guessed responses was a main effect of boundaries, with older boundaries increasing the amount of backward telescoping, but no main effect of actual date or interaction (Figure 1C).
Table 1

Example Stimuli of Low, Medium, and High Knowledge Events From Each Year

\begin{tabular}{|c|c|c|}
\hline Year & $\begin{array}{l}\text { Knowledge } \\
\text { Level }\end{array}$ & Event \\
\hline \multirow[t]{3}{*}{1997} & Low & $\begin{array}{l}\text { Paris court convicts the assassin nick- } \\
\text { named "Carlos the Jackal" }\end{array}$ \\
\hline & Medium & $\begin{array}{l}\text { Fashion designer Gianni Versace is mur- } \\
\text { dered in Miami shooting }\end{array}$ \\
\hline & High & $\begin{array}{l}\text { The film Titanic, directed by James } \\
\text { Cameron, opens in US }\end{array}$ \\
\hline \multirow[t]{3}{*}{1998} & Low & $\begin{array}{l}\text { Pol Pot, leader of Cambodia's infamous } \\
\text { Khmer Rouge movement, dies }\end{array}$ \\
\hline & Medium & $\begin{array}{l}\text { Multi-Olympic gold medalist Florence } \\
\text { Griffith Joyner dies }\end{array}$ \\
\hline & High & $\begin{array}{l}\text { Swissair Flight } 111 \text { crashes off Nova } \\
\text { Scotia coast, killing } 229\end{array}$ \\
\hline \multirow[t]{3}{*}{1999} & Low & $\begin{array}{l}\text { Wen Ho Lee arrested for spying on U.S. } \\
\text { nuclear secrets for China }\end{array}$ \\
\hline & Medium & $\begin{array}{l}\text { Albertan Joseph Faulder executed in } \\
\text { Texas by lethal injection }\end{array}$ \\
\hline & High & $\begin{array}{l}\text { Two students gun down and kill } 13 \text { in } \\
\text { Columbine High School }\end{array}$ \\
\hline \multirow[t]{3}{*}{2000} & Low & $\begin{array}{l}\text { Peace agreement between Ethiopia and } \\
\text { Eritrea ends costly war }\end{array}$ \\
\hline & Medium & $\begin{array}{l}\text { French Concorde crashes after takeoff, } \\
\text { killing all on board }\end{array}$ \\
\hline & High & $\begin{array}{l}\text { Tornado kills } 12 \text { and injures } 140 \text { at Pine } \\
\text { Lake Northern Alberta }\end{array}$ \\
\hline \multirow[t]{3}{*}{2001} & Low & $\begin{array}{l}\text { U.S. submarine collides with Japanese } \\
\text { trawler, killing nine }\end{array}$ \\
\hline & Medium & $\begin{array}{l}\text { Congressional intern Chandra Levy is } \\
\text { first reported missing }\end{array}$ \\
\hline & High & $\begin{array}{l}\text { Edmonton baby Erica Nordby revived } \\
\text { after being found frozen }\end{array}$ \\
\hline
\end{tabular}

Boundary values were likely to be the only numerical information involved when respondents guessed.

In summary, the boundary-effects model predicted a boundary by actual date interaction for nonguessed responses when estimated dates were regressed against actual dates. The associative model, on the other hand, predicted no effect of boundaries. The dual-strategies position predicted that guessed responses would show a main effect of boundaries but no effect of actual date or boundary $X$ actual date interaction.

\section{METHOD}

\section{Participants}

Two hundred University of Alberta students participated in this experiment. The participants were Canadians under 22 years of age, and they received course credit in a procedure lasting approximately $45 \mathrm{~min}$. These participants were randomly assigned to four groups.

\section{Materials}

The stimuli consisted of 64 Canadian and international news events occurring between January 1997 and June 2001. Eight events were selected from 2001 and 14 events from each of the remaining years. The 64 items were distributed as equally as possible across the range. Approximately half these items were political events, and the other half were events such as natural disasters, unusual sporting incidents (e.g., Mike Tyson bites Evander Holyfield's ear) and human-interest stories. 
The events were chosen to be uniquely identifiable and not, on the whole, aspects of larger ongoing stories. In addition, the events were not easily datable because of seasonal factors. Table 1 shows low-, medium-, and high-knowledge examples from each year in the true range.

\section{Procedure}

The news events were serially presented and uniquely randomized for each task and participant. In Task 1, the participants were asked to rate each event according to how much they knew about the event, using a $0-8$ scale where $0=I$ know nothing about this to $8=I$ know a great deal about this. The participants were informed that the " 0 " response equated never having heard of the event before. The participants were informed that the numbers 0 and 8 represented two extreme ends of a scale and that they should consider their responses carefully. This rating scale can be used to compare known events with unknown events. Zero-rated events are assumed to be guessed in the subsequent event-dating task because these events were unknown or unrecognized. ${ }^{1}$ For instance, presented with " 6 yr. old Cuban refugee Elian Gonzalez is returned to Havana," participants who had never heard of Elian Gonzalez would have few options other than to guess. This is because the event is atypical, nonseasonal, and unlikely to be dated using the additional information - "6 yrs old," "Cuban refugee," or "Havana."

In Task 2, the participants were randomly assigned to one of four boundary conditions and were asked to date each event as accurately as possible to the nearest month. These boundaries served as limits on the earliest permissible date estimates. The boundaries' values were January 1997 (true boundary), January 1994, January 1991, and an unbounded condition. In the bounded conditions, the computer program rejected responses outside of the assigned boundaries asked the participant to estimate the same event again. Nonresponses were not permitted in any task. All material and data were computer controlled. This experiment was conducted during October and November 2001.

\section{RESULTS}

Guesses were defined as events receiving a 0 in the knowledge rating task. Although some events were better known than others, there was no significant difference in the number of guesses from each year of the range. Therefore, median estimated dates were calculated for each participant, collapsing over events from each year in the actual range. These medians served as the primary unit of analysis in the following calculations.

\section{All Responses}

Figure 2A presents the pattern of responses when estimated dates are regressed against the actual year. These data are consistent with the pattern of responses predicted by the boundary model. As boundaries recede toward the past, the intercepts decrease and slopes increase. Median estimates were entered into a 4 (boundary) $\times 5$ (actual year) mixed-factors analysis of variance (ANOVA). There was a significant main effect of boundaries $[F(3,980)=$ 21.24, $\left.M S_{\mathrm{e}}=1.46, p<.001\right]$. Post hoc comparisons showed that all bounded conditions were different, although there was no significant difference between the unbounded estimates and those with a 1994 boundary. There was a main effect of actual year $\left[F(4,980)=80.01, M S_{\mathrm{e}}=\right.$ $1.46, p<.001]$, reflecting people's ability to discriminate between events from different years. All years showed significant differences, apart from estimates for events occurring in 2000 and 2001. No significant interaction was detected $\left[F(12,980)=.49, M S_{\mathrm{e}}=0.71, p>.923\right]$.

Although these data are consistent with the boundaryeffects position, evidence for the contrary was also found because unbounded estimates showed a pattern of forward and backward telescoping similar to that of the bounded responses.

\section{Guesses}

Thirty-six percent of knowledge ratings received a 0 indicating that the frequency of guessing was high. Fig-
(A) All Responses

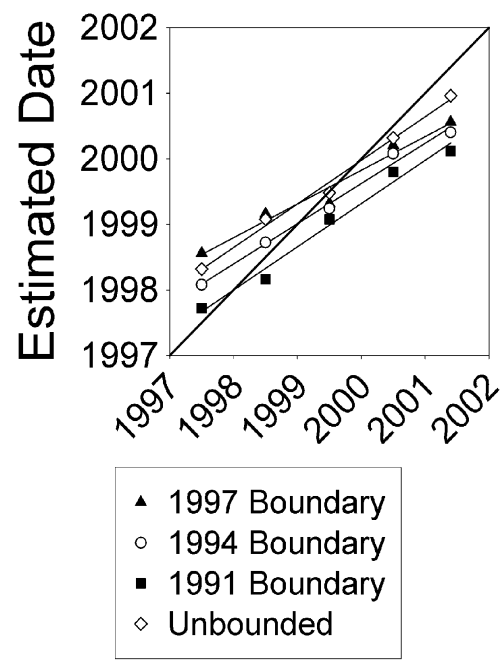

(B) Guessed Responses

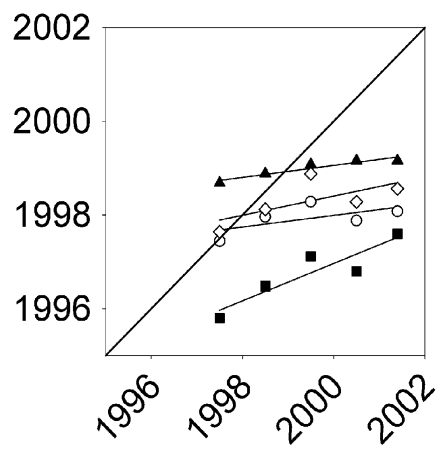

Actual Date
(C) Nonguessed Responses

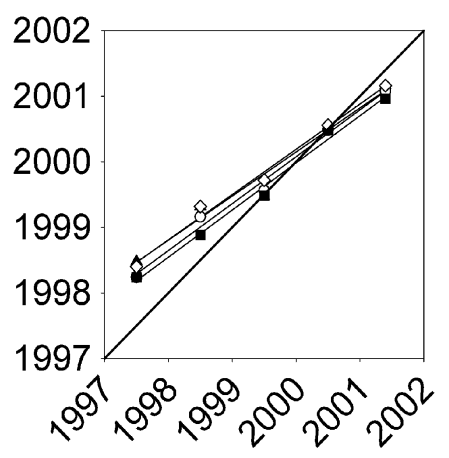

Figure 2. Patterns of response biases for guessed and nonguessed responses. 


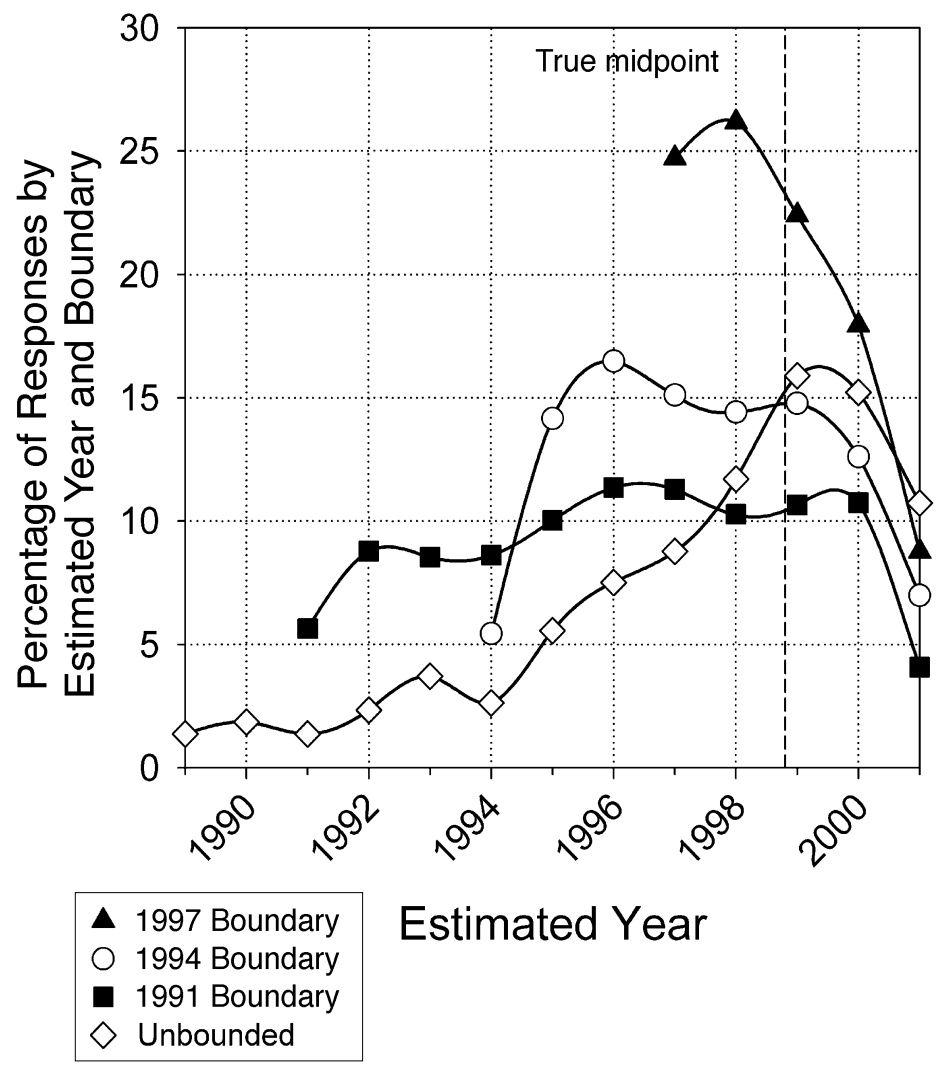

Figure 3. Proportion of guessed responses as a function of estimated year.

ure 3 shows the percentage of guessed responses as a function of estimated year. The proportion of guessed responses, in each condition, predating the middle of the true range, increases as boundaries recede toward the past. Specifically, $67 \%, 75 \%$, and $81 \%$ of guessed responses predated the true midpoint in the 1997 boundary, 1994 boundary, and 1991 boundary conditions, respectively. The only significant result from a 4 (boundary) $\times 5$ (actual year) ANOVA was a main effect of boundaries $\left[F(3,927)=12.32, M S_{\mathrm{e}}=36.57, p<.001\right]$. Guessed estimates from across the range were backward telescoped, and the magnitude of the bias was contingent on boundary information (Figure 2B).

\section{Nonguessed Responses}

The 4 (boundary) $\times 5$ (actual year) ANOVA was repeated with guessed responses omitted from the data set. As expected, the main effect of actual year remained $\left[F(4,980)=46.85, M S_{\mathrm{e}}=3.25, p<.001\right]$. The main effect of boundaries was not significant $[F(3,980)=2.29$, $\left.M S_{\mathrm{e}}=3.25, p>.05\right]$ (Figure 2C). ${ }^{2}$ More importantly, and contrary to the boundary-effects position, no interaction was detected $\left[F(12,980)=0.88, M S_{\mathrm{e}}=3.25, p>.05\right]$. These data demonstrated the type of telescoping effects described in Figure 1B. With guesses removed, the amount of forward telescoping is almost uniform across conditions.
As suggested by the dual-strategies approach, biasing is very different for nonguessed as opposed to guessed responses. Our initial conclusion, that boundaries are the primary cause of forward telescoping, can be rejected. In addition, the elimination of backward telescoping indicates that this bias can be attributed to guessing.

\section{DISCUSSION}

This study determined that guessing of event dates was nonrandom and was sufficiently affected by different boundary information to influence the overall pattern of responses significantly. When all date estimates were analyzed, the boundary-effects model predicted the obtained biases; as boundaries receded in time, forward telescoping decreased. However, an examination of the guessed responses indicated that the reduction in forward telescoping and increase in backward telescoping was almost entirely attributable to respondents guessing the date for unknown events. Because guessing takes into account range information, the tendency for people to estimate unknown events near the midpoint of a range, or earlier, serves to increase backward telescoping of events when boundaries recede. With guessed responses omitted from the analysis, there was no effect of different boundaries on forward telescoping, and backward tele- 
scoping was almost entirely eliminated. This leads us to reject the boundary-dependent explanation of forward telescoping and, necessarily, accept the associative model as the only current alternative.

By understanding the relationship between estimation strategy and estimation biases, we were able to isolate and identify the cause of backward telescoping. We argued that strategy selection depends on people's ability to retrieve relevant information. Apparently, when events are completely unknown (or unrecognized), boundaries provide numerical information and are an important factor in estimating event dates. Guessing is not arbitrary, because people are likely to treat the boundary information as useful and veridical (for a review of communicative maxims, see Schwarz, 1996). The strategy of guessing the midpoint of a range (or earlier) is consistent with two rational assumptions: (1) that people have an intuitive understanding that unknown events are likely to be old because they may have been forgotten (Brown et al., 1985); and (2) that estimating the midpoint limits absolute error when the distribution of actual values is unknown (Huttenlocher et al., 1991).

The study also highlights the fundamental difference between the boundary-effects and associative approaches. The boundary-effects model does not explain the role of inferential information in the biasing of date estimates. Instead, the model relies on the statistical properties in the distribution of people's errors. In contrast, the associative model is an attempt to explain forward telescoping from the standpoint of retrieval, inference, and estimation processes. At the heart of the associative model is a reconstructive process that retrieves and applies potentially relevant information from memory. There is good evidence that reconstruction plays an important role in date estimation (Brown, 1990; Friedman, 1993; Thompson et al., 1988), and Kemp's associative model is the only one that has attempted to simulate this general process. Kemp's (1999) explication of the associative model focused on only one type of potentially relevant temporal information (where dates are stored for episodes related to the target event), one type of memory dynamic, and the exponential loss of information over time. It is reasonable to assume that people forget things over time, but the dating literature indicates that useful temporal information comes in many different forms (e.g., Brown, 1990; Friedman, 1993; Huttenlocher et al., 1988). This fact suggests that the associative model requires further elaboration. Nonetheless, it does seem that forward telescoping occurs because the reconstructive process is likely to retrieve facts that are more recent than the target event. It appears that forgetting and reconstruction combine to produce forward telescoping.

Finally, this study provides additional evidence for the general applicability of the multiple-strategies approach in real-world cognition (Siegler, 1987b). This perspective is characterized by a process that identifies different cognitive strategies and attempts to determine how those strategies affect performance. The emphasis on strate- gies and performance embraces the notion that many processes may describe how respondents estimate numerical values.

\section{REFERENCES}

Baddeley, A., Lewis, V., \& Nimmo-Smith, I. (1978). When did you last . . . ? In M. Gruneberg, P. Morris, \& R. Sykes (Eds.), Practical aspects of memory (pp. 77-83). Orlando, FL: Academic Press.

Blair, E., \& Burton, S. (1987). Cognitive processes used by survey respondents to answer behavioral frequency questions. Journal of Consumer Research, 14, 280-288.

Brown, N. R. (1990). Organization of public events in long-term memory. Journal of Experimental Psychology: General, 119, 297-314.

Brown, N. R. (1995). Estimation strategies and the judgment of event frequency. Journal of Experimental Psychology: Learning, Memory, \& Cognition, 21, 1539-1553.

Brown, N. R. (2002). Real-world estimation: Estimation modes and seeding effects. Psychology of Learning \& Motivation, 41, 321-359.

Brown, N. R., Rips, L. J., \& Shevell, S. K. (1985). The subjective dates of natural events in very-long-term memory. Cognitive Psychology, 17, 139-177.

BROWN, N. R., \& Sincla R, R. C. (1999). Estimating number of lifetime sexual partners: Men and women do it differently. Journal of Sex Research, 36, 292-297.

BuRT, C. D. B. (1992). Retrieval characteristics of autobiographical memories: Event and date information. Applied Cognitive Psychology, 6, 389-404.

BURT, C. D. B., KeMP, S., \& Conway, M. (2001). What happens if you retest autobiographical memory 10 years on? Memory \& Cognition, 29, 127-136.

CRAWLEY, S. E., \& Pring, L. (2000). When did Mrs. Thatcher resign? The effects of aging on the dating of public events. Memory, $\underline{\mathbf{8}}, 111$ 121.

Ferguson, R. P., \& Martin, P. (1983). Long-term temporal estimation in humans. Perception \& Psychophysics, 33, 585-592.

Friedman, W. J. (1993). Memory for the time of past events. Psychological Bulletin, 35, 1-36.

Gentner, D., \& Collins, A. (1981). Studies of inference from lack of knowledge. Memory \& Cognition, 9, 434-443.

Goldstein, D. G., \& GigerenZER, G. (1999). The recognition heuristic: How ignorance makes us smart. In G. Gigerenzer \& P. Todd (Eds.), Simple heuristics that make us smart (pp. 37-57). New York: Oxford University Press.

Huttenlocher, J., Hedges, L. V., \& Duncan, S. (1991). Categories and particulars: Prototype effects in estimating spatial location. Psychological Review, 98, 352-376.

Huttenlocher, J., HEDges, L. V., \& Prohaska, V. (1988). Hierarchical organization in ordered domains: Estimating the dates of events. Psychological Review, 95, 471-484.

KeMP, S. (1993). Bias in dating news and historical events. Acta Psychologica, 86, 69-87.

KEMP, S. (1999). An associative theory of estimating past dates and past prices. Psychonomic Bulletin \& Review, 6, 41-56.

Larsen, S. F., Thompson, C. P., \& Hansen, T. (1996): Time in autobiographical memory. In D. C. Rubin (Ed.), Remembering our past: Studies in autobiographical memory (pp. 129-156). New York: Cambridge University Press.

Lieury, A., Caplain, P., Jacquet, A., \& Jolivet, C. (1979). The contraction of time in dating old events. L'Année Psychologique, 79, 7-22.

Rubin, D. C., \& BADDELEY, A. D. (1989). Telescoping is not time compression: A model of the dating of autobiographical events. Memory \& Cognition, 17, 653-661.

ScHWARZ, N. (1996). Cognition and communication: Judgmental biases, research methods and the logic of conversation. Mahwah, $\mathrm{NJ}$ : Erlbaum.

SIEGLER, R. S. (1987a). The perils of averaging data over strategies: An example from children's addition. Journal of Experimental Psychology: General, 116, 250-264.

SIEGLER, R. S. (1987b). Strategy choices in subtraction. In J. A. Slo- 
boda \& John D. Rogers (Eds.), Cognitive processes in mathematics: Seminar proceedings (Keele Cognition Seminars Vol. 1, pp. 81-106). Oxford: Oxford University Press, Clarendon Press.

Thompson, C. P. (1982). Memory for unique personal events: The roommate study. Memory \& Cognition, 10, 324-332.

Thompson, C. P., SKOwronski, J. J., \& BETZ, A. (1993). The use of partial temporal information in dating personal events. Memory \& Cognition, 21, 352-360.

Thompson, C. P., Skowronski, J. J., Larsen, S. F., \& Betz, A. (Eds.) (1996). Autobiographical memory: Remembering what and remembering when. Mahwah, NJ: Erlbaum.

Thompson, C. P., Skowronski, J. J., \& LeE, D. J. (1988). Telescoping in dating naturally occurring events. Memory \& Cognition, 16, 461468 .

\section{NOTES}

1. Previous studies have used self-reports to identify and measure guessed responses (e.g., strategy menus). However, it is not necessary that respondents can, or do, differentiate between low confidence estimates and guessed responses. In this article, we use an objective method to identify guesses and stimuli whose dates are unlikely to be inferred if the event is unrecognized.

2. The probability of a main effect of boundaries was marginal (.1 $>$ $p>.05)$. However, we consider this result nonmeaningful, as well as nonsignificant, considering the obtained effect size $\left(\eta^{2}=.007\right)$.

(Manuscript received July 28, 2003;

revision accepted for publication October 9, 2003.) 